\title{
Spontaneous optomechanical pattern formation in cold atoms
}

\author{
E. Tesio, G. R. M. Robb, T. Ackemann, W. J. Firth, and G.-L. Oppo \\ ICS, SUPA, and Department of Physics, University of Strathclyde, Glasgow G4 ONG, United Kingdom
}

(Received 6 April 2012; published 10 September 2012)

\begin{abstract}
Transverse pattern formation in an optical cavity containing a cloud of cold two-level atoms is discussed. We show that density modulation becomes the dominant mechanism as the atomic temperature is reduced. Indeed, for low but easily achievable temperatures the internal degrees of freedom of the atoms can be neglected, and the system is well described by treating them as linear dielectric particles. A linear stability analysis predicts the instability threshold and the spatial scale of the emergent pattern. Numerical simulations in two transverse dimensions confirm the instability and predict the spontaneous formation of honeycomb and hexagonal density structures, respectively, for the blue and red detuned cases.
\end{abstract}

DOI: 10.1103/PhysRevA.86.031801

PACS number(s): 42.65.Sf, 37.10.Vz

Pattern formation is defined as the spontaneous emergence of spatiotemporal structures in nonlinear systems driven far from equilibrium [1]. The spatial structure emerges from an initially homogeneous state as a consequence of the interplay between local nonlinearity and spatial coupling mechanisms such as diffusion and diffraction. In optical systems, it is well known that diffraction and optical nonlinearities can induce spatiotemporal structures inside the medium through modulation of populations and coherences, in different media and geometric configurations [2-11].

An additional mechanism for spontaneous self-organization appears in atomic media cold enough for optical forces to overcome thermal effects. Nonlinear optical effects involving the mechanical effect of light on cold atoms are well known and have been predicted and demonstrated in cold atomic gases [12-20] and Bose-Einstein condensates [21-23]. In the context of the collective atomic recoil laser (CARL), atomic bunching along the axis of a high-quality ring cavity has been predicted [12] and observed [13,14]. Self-crystallization of a pencil-shaped cloud of cold atoms has been shown to lead to high-order nonlinearities [15] and superradiance [16]. Collective scattering from a two-level sample illuminated transversally to the cavity axis can also lead to a runaway instability where a standing wave develops in the cavity and is self-sustained by a two-dimensional (2D) density grating generated by the interference of the pump and the cavity field $[18,19]$. Experimental evidence was found in Refs. [20,22,23]. In these schemes, however, the periodicity is crucially determined by the light wavelength, and the emerging density modulation is fully imposed by the external geometry. In contrast, we consider here a spontaneous instability in the plane orthogonal (transverse) to a single pump wave, where the resulting stable patterns are determined by a combination of nonlinearity and tunable diffractive phase shifts. The transverse plane is isotropic around the pump axis and hence the symmetry of the emerging pattern is determined by spontaneous symmetry breaking. It turns out that spontaneous hexagons and honeycombs are selected at threshold.

In soft matter systems, density-driven nonlinear mechanisms have been shown to give rise to self-focusing [24], four-wave mixing [25], bistability [26], optical binding [27], formation of soliton arrays in optical waveguides [28], and nonlinear backscattering [29,30]. In these studies the formation of two-dimensional structures arising from spontaneous symmetry breaking in the plane orthogonal to the pump axis and due to optomechanical forces has not been addressed and is the main topic of this Rapid Communication.

The possibility of using density redistribution effects in cold atomic gases for spontaneous filamentation in a matter wave has been proposed in Ref. [31]. For counterpropagating beams, a lowering in the threshold for transverse selforganization on the focusing side of the nonlinearity has been proposed [32,33]. Experimental evidence of the formation of transverse structures in cold atomic media has also been found in Refs. [34,35]. These previous studies typically emphasize the interplay between mechanical density redistribution effects and the nonlinearities arising from the internal degrees of freedom of the atom, and often involve multiple optical beams forming wavelength-scale lattices, and perhaps also optical polarization effects related to the multilevel quantum structure of the atoms.

In contrast, we consider a very simple, and hence general, system of ground-state atoms interacting with a single coherent optical field. The linear dielectric response of the atoms, which is responsible for the refractive index of the cloud, means that the atoms will move up or down any transverse gradient in the optical field. In turn, the refractive effects of nonuniform atomic density will lead to phase gradients, and thus eventually intensity nonuniformities, in the optical field. As we will show, this simple mechanism readily produces positive feedback, and thus transverse instability, independently of the sign of the atomic response. We illustrate this very general instability mechanism for the case of a Doppler cooled two-level atom cloud within a planar ring cavity driven by a monochromatic plane wave optical field. This simple fundamental atom-field coupling is, of course, already present in all the above-mentioned experiments, and in many of the models. Its importance has not previously been apparent because of the complexity of the systems considered. We believe that the results of our simple model will be important for the interpretation of these more complex experiments and models, and hence for future progress in cold-atom optics.

We consider a sample of $N$ identical, noninteracting twolevel atoms inserted in a planar ring cavity. Optical molasses are assumed to cool the sample at a temperature $T$ (see Fig. 1). We allow for a redistribution of the cloud density in the form $N(\mathbf{x}, t)=N_{0} n(\mathbf{x}, t)$, where $N_{0}$ is the uniform spatial density of 


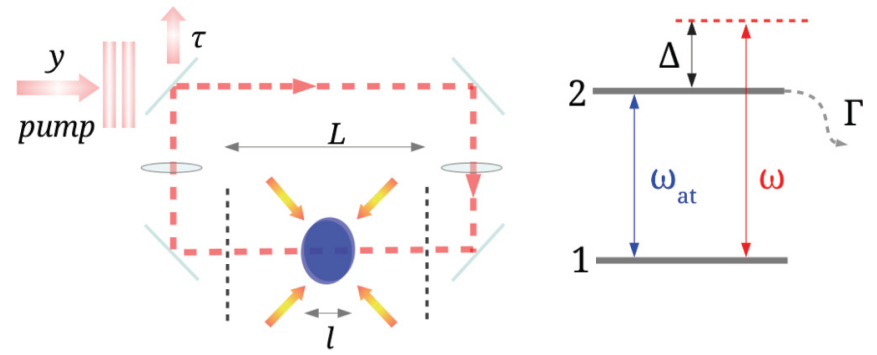

FIG. 1. (Color online) A sample of two-level atoms with thickness $l$ and density $N_{0}$ is inserted in a planar ring cavity of effective length $L$ and laser cooled at a temperature $T$. $L$ can be controlled by adjustment of the intracavity lenses, and is therefore distinct from the physical cavity length. A plane wave of amplitude $y$ and frequency $\omega$, detuned from the resonance $\omega_{\text {at }}$ by $\Delta$, pumps the medium in the cavity. The mirror transmittivity is $\tau$.

the sample and $n(\mathbf{x}, t)$ encodes a spatial density modulation. The cloud susceptibility is cast in the form $\chi=N(\mathbf{x}, t) \chi_{\mathrm{e}}(\mathbf{x}, t)$, where $N$ accounts for the density redistribution effects and $\chi_{\mathrm{e}}$ represents the electronic susceptibility. Adiabatic elimination of populations and coherences in the optical Bloch equations of a two-level system are known to produce an intensitydependent electronic response of the form $\chi_{\mathrm{e}}=\chi_{\mathrm{e}}\left(|f(\mathbf{x}, t)|^{2}\right)$, where $f$ represents the amplitude of the optical field [4]. The cloud polarization $\chi f$ then acts as a nonlinear source term in the field wave equation, which can be written in the slowly varying envelope, rotating wave, paraxial, and mean-field approximations as [4]

$$
\dot{f}=-(1+i \theta) f+y-\frac{\tilde{\gamma} n}{1+\frac{|f|^{2}}{1+\Delta^{2}}} f+i \nabla_{\perp}^{2} f .
$$

Equation (1) contains only adimensional quantities, with the field $f$ rescaled to the saturation intensity at resonance and the time normalized to the cavity losses (see the term $-f$ ). $\Delta$ is the light-atom detuning (in units of the coherence decay rate $\Gamma / 2$ ), while $\nabla_{\perp}^{2}=\partial_{x}^{2}+\partial_{y}^{2}$ denotes the transverse Laplacian and describes diffraction. The cavity imposes a linear phase shift governed by the cavity detuning $\theta$, and is pumped by a plane wave of (normalized) amplitude $y$. Definitions are chosen so that $\Delta>0(\Delta<0)$ corresponds to blue (red) detuned beams and thus to self-focusing (defocusing) nonlinearities. The strength of the complex susceptibility is $\tilde{\gamma}=2 C(1+i \Delta) /\left(1+\Delta^{2}\right)$, with absorption and dispersion captured by its real and imaginary parts, respectively. The cooperativity parameter $C$ contains the dependence on the sample density $N_{0}$ and the mirror transmittivity $\tau$. Spatial coordinates are normalized to the diffraction length $\sqrt{a}=$ $\sqrt{\lambda L / 4 \pi \tau}$, where $\lambda$ is the radiation wavelength and $L$ the effective cavity length (see Fig. 1). Note that terms in the Maxwell equations varying as $\dot{n}, \ddot{n}$ are neglected in deriving Eq. (1).

If $n$ is considered uniform, Eq. (1) becomes a standard model of cavity nonlinear optics, showing optical bistability and pattern formation for appropriate parameter choices. When the nonlinear term in Eq. (1) is neglected, no instability is possible for constant $n$, and the role of the atoms is simply to modify the cavity losses and resonance frequencies through the complex linear susceptibility. If we allow the density to enter as a dynamical variable, however, the system behavior becomes qualitatively different. To proceed, it is necessary to specify an equation for the dynamics of the density modulation $n$, which is coupled back to the field through the action of optical forces. In the large detuning limit analyzed here scattering forces are negligible, and the cloud is subject only to the dipole potential $U_{\text {dip }}=\left(\hbar \Gamma \Delta|f|^{2}\right) / 4\left(1+\Delta^{2}\right)$. In the presence of optical molasses the atoms of the cloud experience a strong momentum damping and undergo spatial diffusion with a coefficient $D$, which is dependent on the molasses parameters [36]. A continuity equation for the density distribution can be written as $[33,37]$

$$
\dot{n}=\sigma D \nabla_{\perp} \cdot\left[n \nabla_{\perp} \frac{|f|^{2}}{1+\Delta^{2}}\right]+D \nabla_{\perp}^{2} n, \quad \sigma=\frac{\hbar \Gamma \Delta}{4 k_{\mathrm{B}} T} .
$$

We recognize the first term on the right-hand side of Eq. (2) as the divergence of a drift current originating from the transverse dipole forces, potentially leading to nonuniform density, while the second term, diffusion, opposes such nonuniformities. The parameter $\sigma$ characterizes the relative strength of these opposing tendencies. We stress that the presence of the molasses is needed in order to damp the motion imposed by dipole forces, as it was also demonstrated to be beneficial for the self-organization in CARL systems [14]. We see from Eq. (2) that density modulations are favored by large detuning and, importantly, by low temperature. In the limit of high temperatures $(\sigma \rightarrow 0)$ diffusion drives the atomic distribution towards the homogeneous state $(n=1)$, and the model reproduces the hot-atoms situation. The stationary state for the density modulation is given by the equilibrium distribution $[33,37]$

$$
n_{\mathrm{eq}}(\mathbf{x})=\frac{V \exp \left(-U_{\mathrm{dip}} / k_{\mathrm{B}} T\right)}{\int_{V} d \mathbf{x} \exp \left(-U_{\mathrm{dip}} / k_{\mathrm{B}} T\right)},
$$

where $V$ is the sample volume.

Equations (1) and (2) describe the coupled dynamics of the intracavity field and the two-level sample when both electronic and optomechanical effects are present. This kind of system, and the role played by the temperature, have been addressed for the arrangement of two counterpropagating beams in Refs. [32,33]. Here we study the much simpler situation of a unidirectional beam and, moreover, negligible electronic nonlinearity, showing that optomechanical effects alone are capable of providing a pattern-forming instability through density redistributions. For large detuning $(|\Delta| \gg 1)$ absorption is negligible compared to dispersion. If we also neglect the nonlinear dispersive response, the system of Eqs. (1) and (2) reduces to

$$
\begin{aligned}
& \dot{f}=-(1+i \theta) f+y-i \gamma n f+i \nabla_{\perp}^{2} f, \\
& \dot{n}=\sigma D \nabla_{\perp} \cdot\left[n \nabla_{\perp} \frac{|f|^{2}}{1+\Delta^{2}}\right]+D \nabla_{\perp}^{2} n,
\end{aligned}
$$

where $\gamma=\operatorname{Im}[\tilde{\gamma}]=2 C \Delta /\left(1+\Delta^{2}\right)$ accounts for linear dispersion and nonlinear terms in $|f|^{2} /\left(1+\Delta^{2}\right)$ have been neglected. We remark that this limit can be experimentally feasible. For a $10-\mathrm{mm}$-thick sample of ${ }^{85} \mathrm{Rb}$ with density $N_{0}=7 \times 10^{10}$ atoms $/ \mathrm{cm}^{3}$ at a temperature $T=300 \mu \mathrm{K}$, interacting with a laser beam detuned by $|\Delta|=100$ linewidths 
from the $D_{2}$ line (transition wavelength $780 \mathrm{~nm}$ ) and mirror transmittivity of $10 \%$, for instance, one obtains $C \simeq 225$ and $|\sigma| \simeq 25$, and we will find that the electronic nonlinearity is indeed small at the threshold for density-driven pattern formation. To demonstrate this, we perform a linear stability analysis of the system (4). The flat, stationary state of Eqs. (4) is given by $f_{\mathrm{s}}=y[1+i(\theta+\gamma)]^{-1}, n_{\mathrm{s}}=1$. We perturb this flat solution as $f=f_{\mathrm{s}}+\delta f(\mathbf{x}, t), n=1+\delta n(\mathbf{x}, t)$, where $\mathbf{x}$ is the transverse coordinate, and linearize the system (4). Looking for static instabilities, we write the perturbations in the form $\sim e^{i \mathbf{q} \cdot \mathbf{x}} e^{\lambda(\mathbf{q}) t}$ (with $\lambda$ real) and impose the condition of marginal stability, i.e., $\lambda(\mathbf{q})=0$. A threshold condition is found for the control parameter $I=\left|f_{\mathrm{s}}\right|^{2}$, which depends only on the modulus $q$ of the transverse wave vector due to rotational symmetry:

$$
\begin{aligned}
q_{\mathrm{c}, \mathrm{OM}}^{2} & =1-(\theta+\gamma), \\
I^{\mathrm{OM}}\left(q^{2}\right) & =\frac{\left(1+\Delta^{2}\right)\left[1+\left(\theta+\gamma+q^{2}\right)^{2}\right]}{2 \sigma \gamma\left(\theta+\gamma+q^{2}\right)} .
\end{aligned}
$$

Here $\mathbf{q}_{\mathrm{c}, \text { OM }}$ denotes the critical wave vector of the system (4), i.e., the first transverse mode to become excited when increasing the control parameter. At difference from Refs. [18,19], the spatial instability is truly spontaneous since there are no explicitly modulated terms at $\mathbf{q}_{\mathrm{c}, \mathrm{OM}}$ in Eqs. (4). The critical wave number can be controlled by varying the cavity detuning $\theta$ : We set $\theta+\gamma=-1(\theta \simeq-5.5)$, which gives $q_{\mathrm{c}, \mathrm{OM}}^{2}=2 . I^{\mathrm{OM}}\left(q^{2}\right)$ represents the value of intensity such that a perturbation with wave number $\mathbf{q}$ becomes unstable, $\lambda(\mathbf{q})=0$. We find the minimum threshold $I_{\mathrm{th}}^{\mathrm{OM}} \simeq 88.9$ for our choice of parameters: The low-excitation assumption is therefore confirmed since $I_{\text {th }}^{\mathrm{OM}} /\left(1+\Delta^{2}\right) \simeq 0.009 \ll 1$. Figure 2 shows the threshold curves $I\left(q^{2}\right)$ for our choice of parameters. Figure 2(a) compares the threshold for the purely optomechanical system, Eq. (5b) (solid line), with that of the full system involving both electronic and mechanical effects, Eqs. (1) and (2), for blue (blue dashed line) and red (red dotted-dashed line) detuning. The minimum thresholds for the full model are found to be $I_{\text {th }} \simeq 90.8$ (blue detuning)
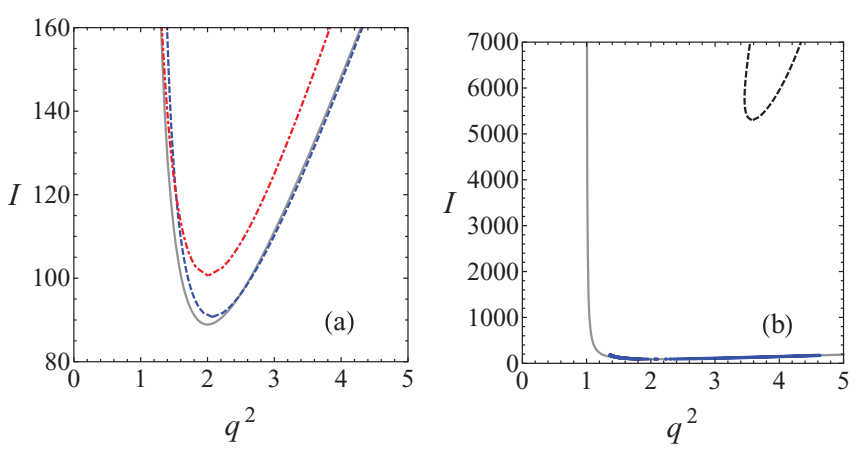

FIG. 2. (Color online) Left: Pattern-formation threshold curves for Eqs. (4) (solid line) and for the full system Eqs. (1) and (2) for blue (dashed line) and red (dashed-dotted line) detuning. $|\Delta|=$ 100, $|\sigma|=25, C=225$, and $\theta+\gamma=-1$. Right: On a much larger intensity scale, thresholds for the purely mechanical system (solid line), the complete system (blue dots), and the blue-detuned case of the saturable Kerr model (dashed line) without density modulation. and $I_{\text {th }} \simeq 100.7$ (red detuning). The critical wave numbers are $q_{\mathrm{c}}^{2} \simeq 2.07$ (blue detuning) and $q_{\mathrm{c}}^{2} \simeq 2.01$ (red detuning). The instability behavior of the full system is clearly dominated by the optomechanical modulation of the atomic density, with electronic effects negligible in first approximation. Further evidence is displayed in Fig. 2(b), which compares the thresholds for the purely mechanical system of Eqs. (4) (solid line) with that for a saturable Kerr medium with no density redistribution effects, i.e., a hot two-level medium (dashed line), which is more than two orders of magnitude greater (for blue detuning, $\Delta=100$ : there is no hot-atom instability for red detuning for our choice of $\theta$ ).

The instability threshold ( $5 b)$ is independent of the sign of the detuning, as positively (negatively) polarized particles are pushed towards the maxima (minima) of the field intensity. The former (latter) case corresponds to the case of suspended dielectric particles of higher (lower) index than the medium. The value of the temperature is crucial in determining the interplay between the optomechanical and the electronic nonlinear mechanisms. The parameter $\sigma$ represents in fact the ratio between the dipole energy $\hbar\left(\omega-\omega_{\mathrm{at}}\right)$ and the thermal energy $k_{\mathrm{B}} T$. For large $|\sigma|$, electronic nonlinearities due to the transition saturation become negligible close to the instability threshold, which can thus be interpreted as due to optomechanical effects alone (see Fig. 2). Although $|\sigma|$ could in principle be increased to very high values by lowering the temperature, our parameters do not require subDoppler temperatures, because the large detuning weakens the electronic response relative to the optomechanical one. We note that the dependence of the instability threshold from detuning, temperature, and density has a universal optomechanical character (see for example the low-finesse cavity case in Ref. [19]).

Numerical simulations in two transverse dimensions have been performed to test the predictions obtained from the linear analysis. Equations (1), (2), and (4) have been integrated using a second-order Crank-Nicholson method, with the density dynamics given by Eq. (3). Periodic boundary conditions are imposed over a domain of five critical wavelengths $\lambda_{\mathrm{c}}=$ $2 \pi / q_{\mathrm{c}}$ in each transverse direction. The transverse domain is discretized using a square grid of $128 \times 128$ points, and time is discretized with step $d t=2 \times 10^{-3}$. Figure 3 presents results of numerical integration in 2D of the simple system (4), for the same parameters as Fig. 2, for both signs of $\sigma$ and $I=95$, about $7 \%$ above threshold. The optomechanical nonlinear mechanism leads in both cases to the formation of hexagonal structures, as is usual in systems with intensity-dependent cubic nonlinearities [38]. The insets in the left panel of Fig. 3 show the six spontaneously generated sidebands in Fourier space (far-field patterns). Different orientations of the sidebands are obtained after subsequent realizations of the numerical simulations as a result of spontaneous symmetry breaking. The distance of the sidebands from the center is determined by the critical wave number [see by Eq. (5a)]. Note that the intensity pattern is bright hexagons in both cases, but the density structure is of honeycomb type for blue detuning. This is because the linear refractive index of the atom cloud is less than unity, so that a "hole" in the cloud has a relatively high index, and so can guide light. We thus interpret the upper panels of Fig. 3 as a self-organized hexagonal 

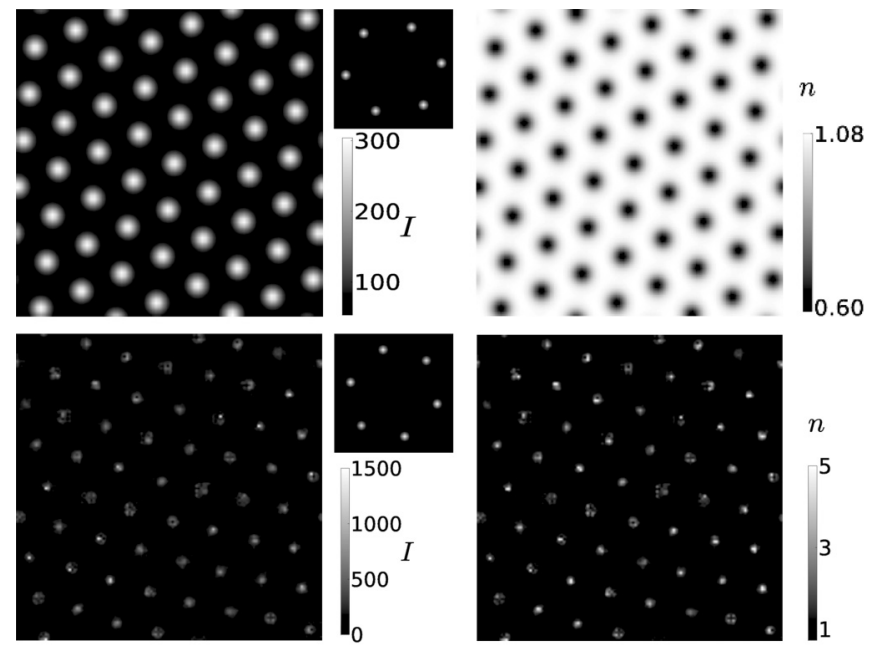

FIG. 3. Optomechanical hexagon formation at about $7 \%$ above threshold. Parameters are as in Fig. 2, with the blue-detuned case in the top panels and the red-detuned case in the bottom panels. Field intensity is depicted on the left, and the corresponding density modulation on the right. The insets in the left panel show the six spontaneously generated sidebands in the far-field pattern. The Fourier mode at zero spatial frequency in the center (defined by the pump and cavity axis) is removed for clarity. The transverse coordinate is rescaled to the diffraction length $\sqrt{a}$, which is of the order of $100 \mu \mathrm{m}$ for a cavity length of few centimeters and a mirror transmittivity of $10 \%$. The box size is $\sim 22$ diffraction lengths. Density structures form according to the values of detuning and temperature-see Eq. (3).

network of waveguides formed by the expulsion of atoms from the guided beams. Since the overlap of light and atoms is reduced in the fully formed pattern the optomechanical nonlinearity has a strong effective saturation, and this pattern is very stable. For red detuning, in contrast, atoms and light attract each other, so that the light is now guided by atomic filaments of high density (lower panels of Fig. 3). As evidenced by the variation of the amplitude of the peaks in Fig. 3 (lower panel), here the interaction is enhanced by the pattern formation, and it is perhaps unsurprising that the stability of the pattern is much poorer for red detuning, though detailed investigation of stability issues is beyond the scope of this Rapid Communication.

As mentioned above, the critical wave number at threshold of Eq. (5a) can be tuned by varying the cavity detuning, i.e., by a change of cavity length on the wavelength scale, with a corresponding variation of the threshold. Macroscopic changes of the cavity length provide instead the overall scale for the emerging patterns via the diffraction parameter $a$. With a view to experimental observation of optomechanical patternforming instabilities, we note that an effective cavity length $L$ of a few centimeters would lead to pattern scales of the order of $\lambda_{\mathrm{c}} \sim 100 \mu \mathrm{m}$, requiring beam diameters of at least $\sim 500 \mu \mathrm{m}$ for the development of a well-defined transverse structure. For standard $\mathrm{Rb}$ molasses at temperatures of $T \sim 100 \mu \mathrm{K}$ the threshold intensity is of order $100 \mathrm{~mW} / \mathrm{cm}^{2}$, so that $2 \mathrm{~mW}$ of intracavity power should be ample to generate optomechanical hexagons.

We have investigated a mechanism for spontaneous optical pattern formation in the transverse plane, dominated by density modulation effects due to dipole forces exerted by light on a cold atomic medium. By considering a very simple ring-cavity configuration we have been able to isolate and highlight the role and importance of optomechanical effects in light-atom interaction at low temperatures. The threshold and the spatial scale of the resulting patterns have been confirmed by numerical simulations in two transverse dimensions. The required atomic temperatures, optical densities, and intensities are well within experimental capabilities, and indeed we suggest that transverse density modulation effects may well be present in previous studies involving more complex configurations. Our simulations predict the formation of self-organized hexagonal waveguiding filaments, the filaments being atom poor or atom rich depending on the optical frequency. As for other systems which form hexagonal patterns, we would expect the existence of stable single filaments, i.e., localized states or dissipative solitons.

We thank R. Martin for useful discussions. Financial support from the Leverhulme Trust (Research Grant No. F/00273/0) and the EPSRC (for G.R.M.R., Grant No. $\mathrm{EP} / \mathrm{H} 049339)$ is gratefully acknowledged.
[1] M. C. Cross and P. C. Hohenberg, Rev. Mod. Phys. 65, 851 (1993).

[2] G. Grynberg, E. Le Bihan, P. Verkerk, P. Simoneau, J. R. R. Leite, D. Bloch, S. Le Boiteux, and M. Ducloy, Opt. Commun. 67, 363 (1988).

[3] L. A. Lugiato and R. Lefever, Phys. Rev. Lett. 58, 2209 (1987).

[4] L. A. Lugiato and C. Oldano, Phys. Rev. A 37, 3896 (1988).

[5] G. L. Lippi, T. Ackemann, L. M. Hoffer, and W. Lange, Chaos Solitons Fractals 4, 1419 (1994); 4, 1433 (1994).

[6] G. Giusfredi, J. F. Valley, R. Pon, G. Khitrova, and H. M. Gibbs, J. Opt. Soc. Am. B 5, 1181 (1988).

[7] W. J. Firth, J. Mod. Opt. 37, 151 (1990).

[8] G. D’Alessandro and W. J. Firth, Phys. Rev. Lett. 66, 2597 (1991); Phys. Rev. A 46, 537 (1992).
[9] T. Ackemann and W. Lange, Phys. Rev. A 50, R4468 (1994); Appl. Phys. B 72, 21 (2001).

[10] R. Neubecker, G.-L. Oppo, B. Thuering, and T. Tschudi, Phys. Rev. A 52, 791 (1995).

[11] C. Denz, M. Shwab, and C. Weilnau, Transverse Pattern Formation in Photorefractive Optics (Springer, Berlin, 2003).

[12] R. Bonifacio and L. De Salvo, Nucl. Instrum. Methods Phys. Res., Sect. A 341, 360 (1994).

[13] C. von Cube, S. Slama, D. Kruse, C. Zimmermann, Ph. W. Courteille, G. R. M. Robb, N. Piovella, and R. Bonifacio, Phys. Rev. Lett. 93, 083601 (2004).

[14] S. Slama, S. Bux, G. Krenz, C. Zimmermann, and Ph. W. Courteille, Phys. Rev. Lett. 98, 053603 (2007). 
[15] J. A. Greenberg and D. J. Gauthier, Eur. Phys. Lett. 98, 24001 (2012).

[16] J. A. Greenberg and D. J. Gauthier, Phys. Rev. A 86, 013823 (2012).

[17] G. L. Gattobigio, F. Michaud, J. Javaloyes, J. W. R. Tabosa, and R. Kaiser, Phys. Rev. A 74, 043407 (2006).

[18] P. Domokos and H. Ritsch, Phys. Rev. Lett. 89, 253003 (2002).

[19] J. K. Asbóth, P. Domokos, H. Ritsch, and A. Vukics, Phys. Rev. A 72, 053417 (2005).

[20] A. T. Black, H. W. Chan, and V. Vuletić, Phys. Rev. Lett. 91, 203001 (2003).

[21] S. Inouye, A. P. Chikkatur, D. M. Stamper-Kurn, J. Stenger, D. E. Pritchard, and W. Ketterle, Science 285, 571 (1999).

[22] D. Schneble, Y. Torii, M. Boyd, E. W. Streed, D. E. Pritchard, and W. Ketterle, Science 300, 475 (2003).

[23] K. Baumann, C. Guerlin, F. Brennecke, and T. Esslinger, Nature (London) 464, 1301 (2010).

[24] A. Askhin, J. M. Dziedzic, and P. W. Smith, Opt. Lett. 7, 276 (1982).

[25] P. W. Smith, A. Ashkin, and W. J. Tomlinson, Opt. Lett. 6, 284 (1981).

[26] P. W. Smith, A. Ashkin, J. E. Biorkholm, and D. J. Eilenberger, Opt. Lett. 10, 131 (1984).
[27] M. M. Burns, J.-M. Fournier, and J. A. Golovchenko, Science 249, 749 (1990).

[28] P. J. Reece, E. M. Wright, and K. Dholakia, Phys. Rev. Lett. 98, 203902 (2007).

[29] G. R. M. Robb and B. W. J. McNeil, Phys. Rev. Lett. 90, 123903 (2003); Phys. Rev. E 67, 025603 (2003).

[30] S. M. Wiggins, G. R. M. Robb, B. W. J. McNeil, D. R. Jones, D. A. Jaroszynski, and S. J. Jamieson, J. Mod. Opt. 49, 997 (2002).

[31] M. Saffman, Phys. Rev. Lett. 81, 65 (1998).

[32] G. A. Muradyan, Y. Wang, W. Williams, and M. Saffman, in Trends in Optics and Photonics (TOPS), Vol. 80, Nonlinear Guided Waves and Their Applications, OSA Technical Digest (OSA, Washington, D.C., 2005), paper ThB29.

[33] M. Saffman and Y. Wang, Lect. Notes Phys. 751, 361 (2008).

[34] J. A. Greenberg, B. L. Schmittberger, and D. J. Gauthier, Opt. Express 19, 22535 (2011).

[35] W. Guerin, F. Michaud, and R. Kaiser, Phys. Rev. Lett. 101, 093002 (2008).

[36] T. W. Hodapp, C. Gerz, C. Furtlenher, C. I. Westbrook, W. D. Phillips, and J. Dalibard, Appl. Phys. B 60, 135 (1995).

[37] K. M. O’Hara, S. R. Granade, M. E. Gehm, and J. E. Thomas, Phys. Rev. A 63, 043403 (2001).

[38] W. J. Firth, A. J. Scroggie, G. S. McDonald, and L. A. Lugiato, Phys. Rev. A 46, 3609R (1992). 